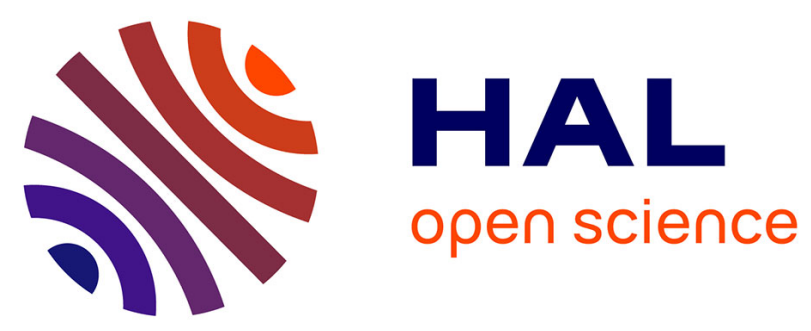

\title{
Three-dimensional numerical simulation of the interaction between natural convection and radiation in a differentially heated cavity in the low Mach number approximation
}

Gilles Scarella, Gilbert Accary, Sofiane Meradji, Dominique Morvan, Oleg

Bessonov

\section{To cite this version:}

Gilles Scarella, Gilbert Accary, Sofiane Meradji, Dominique Morvan, Oleg Bessonov. Threedimensional numerical simulation of the interaction between natural convection and radiation in a differentially heated cavity in the low Mach number approximation. ICHMT International Symposium on Advances in Computational Heat Transfer, May 2008, Marrakech, Morocco. hal-02756802

\section{HAL Id: hal-02756802 \\ https://hal.inrae.fr/hal-02756802}

Submitted on 3 Jun 2020

HAL is a multi-disciplinary open access archive for the deposit and dissemination of scientific research documents, whether they are published or not. The documents may come from teaching and research institutions in France or abroad, or from public or private research centers.
L'archive ouverte pluridisciplinaire HAL, est destinée au dépôt et à la diffusion de documents scientifiques de niveau recherche, publiés ou non, émanant des établissements d'enseignement et de recherche français ou étrangers, des laboratoires publics ou privés. 


\title{
THREE-DIMENSIONAL NUMERICAL SIMULATION OF THE INTERACTION BETWEEN NATURAL CONVECTION AND RADIATION IN A DIFFERENTIALLY HEATED CAVITY IN THE LOW MACH NUMBER APPROXIMATION
}

\author{
Gilles Scarella ${ }^{1}$, Gilbert Accary ${ }^{2, \S}$, Sofiane Meradji ${ }^{3}$, Dominique Morvan ${ }^{3,4}$ and Oleg Bessonov ${ }^{5}$ \\ ${ }^{1}$ I.N.R.A. Unité de Recherches Forestières Méditerranéennes, \\ UR629, Domaine de Saint-Paul, Site Agroparc, F-84914, Avignon, France \\ ${ }^{2}$ Holly-Spirit University of Kaslik, Faculty of Science and Computer Engineering, \\ Department of Science and Technology, B.P. 446, Jounieh, Lebanon \\ ${ }^{3}$ Laboratoire MSNM-GP, UMR 6181 CNRS, I.M.T. La Jetée, Technopôle de Château-Gombert, \\ 38, rue Frédéric Joliot Curie, 13451 Marseille Cedex 20, France \\ ${ }^{4}$ Université de la Méditerranée, UNIMECA, \\ 60, rue Frédéric Joliot Curie, 13453 Marseille Cedex 13, France \\ ${ }^{5}$ Institute for Problems in Mechanics of Russian Academy of Sciences, \\ 101, Vernadsky Avenue, 119526 Moscow, Russia \\ ${ }^{\S}$ Correspondence author: Fax: +9619600901 Email: gilbertaccary@usek.edu.lb
}

\begin{abstract}
Many studies have been devoted to the interaction between natural convection and radiation heat transfer in a differentially heated cavity. This problem has already been treated using the Boussinesq approximation. The main purpose of this study is to extend this interaction to the low Mach number approximation (in 3D), for both transparent and participating media. The NavierStokes and energy equations written for an ideal gas are solved using a finite volume method, while the discrete ordinates method is used to solve the radiation transfer equation. The coupling between the energy equation and the radiation transfer is done by adding an additional source term in the energy equation and via the radiation heat exchange between the surfaces bounding the computation domain. The work is first validated using the Boussinesq approximation mainly by investigating the distribution of the heat flux on the hot isothermal wall. Then some simulations are presented highlighting the differences between the low Mach number and the Boussinesq approximations.
\end{abstract}

\section{NOMENCLATURE}

$L \quad$ cubic enclosure length, [m]

$N \quad$ mesh size in a space direction

$n \quad$ normal vector to a surface

$P_{t h} \quad$ thermodynamic pressure, $[\mathrm{Pa}]$

$Q_{\text {rad }} \quad$ radiative heat flux, [W.m $\left.{ }^{-2}\right]$

$Q^{*} \quad$ dimensionless total heat flux, $-L(\partial T / \partial n) /\left(T_{h}-T_{c}\right)+L Q_{\text {rad }} . n / k_{0}\left(T_{h}-T_{c}\right)$

$R \quad$ ideal gas constant, $\left[\mathrm{J} \cdot \mathrm{Kg}^{-1} \cdot \mathrm{K}^{-1}\right.$ ]

$s \quad$ discrete ordinate

$T^{*} \quad$ dimensionless temperature, $\left(T-T_{c}\right) /\left(T_{h}-T_{c}\right)$ 


\section{Subscripts and superscripts}

$0 \quad$ reference value

c cold boundary

$h$ hot boundary

rad radiative

* dimensionless

\section{Dimensionless groups}

$P l \quad$ Planck number, $k_{0}\left(T_{h}-T_{c}\right) / L \sigma_{B} T_{c}^{4}$

$\operatorname{Pr} \quad$ Prandtl number, $\mu_{0} C_{P 0} / k_{0}$

$R a \quad$ Rayleigh number, $g \rho_{0}{ }^{2} L^{3}\left(T_{h}-T_{c}\right) \operatorname{Pr} / T_{0} \mu_{0}^{2}$

$T_{0}{ }^{*} \quad$ reference temperature ratio, $T_{c} /\left(T_{h}-T_{c}\right)$

\section{Symbols}

$\nabla$. divergence operator, $\partial / \partial x+\partial / \partial y+\partial / \partial z$

\section{INTRODUCTION}

The numerical simulation of fluid flow, with high temperature heat transfers in participating media, is one of the most difficult problems in fluid mechanics. To solve this problem, one needs to manage at least two non-linear phenomena: the convective term in the generalized Navier-Stokes equations (NSE) (continuity, momentum, energy) and the black body emission in the energy and radiation transfer equation (RTE). The numerical resolution of the RTE, requires an additional computational effort, due to the directional nature of the RTE that must be solve along an optical path.

The main purpose of this paper is the analysis of natural convection phenomenon coupled with radiation heat transfer in a three-dimensional differentially heated cavity. Flows in a confined geometry, associated with heat transfer phenomena are commonly encountered in many practical situations. Because of the simplicity of the geometry and the easy way to fix the boundary conditions, this problem has been widely studied and represents a simplified version of more practical scenarios. For the same reasons mentioned just above, it constitutes also a very interesting benchmark problem that is very often used to validate new numerical methods.

Following the previous study of Colomer [2004], this problem has been solved using a dimensionless formulation and for a given set of Rayleigh and Planck numbers, which are the relevant physical parameter governing the convective heat transfer in the cavity. Both nonparticipating and participating media have been investigated. For the latter, several values of the optical thickness were considered. The analysis was limited to the case for which all the walls have the same emissivity. Code validation was performed in the case of Boussinesq assumption and then the analysis has been extended to the low Mach number approximation, thus allowing dealing with large temperature gaps for which the radiative effects are relevant and to which little attention has been paid by the heat transfer community.

\section{CONFIGURATION: DIFFERENTIALLY HEATED CAVITY}

The flow and the heat transfer in a cubic enclosure of length $L$ are analyzed, considering either a transparent or a participating, gray, purely absorbing (non-scattering) homogeneous media. As depicted in Fig. 1, the west wall is maintained isothermal at the temperature $T_{h}$, the east one is 
maintained isothermal at the temperature $T_{c}$ with $T_{h}>T_{c}$, while the remaining four walls are adiabatic. The walls of the cavity have been treated as black bodies.

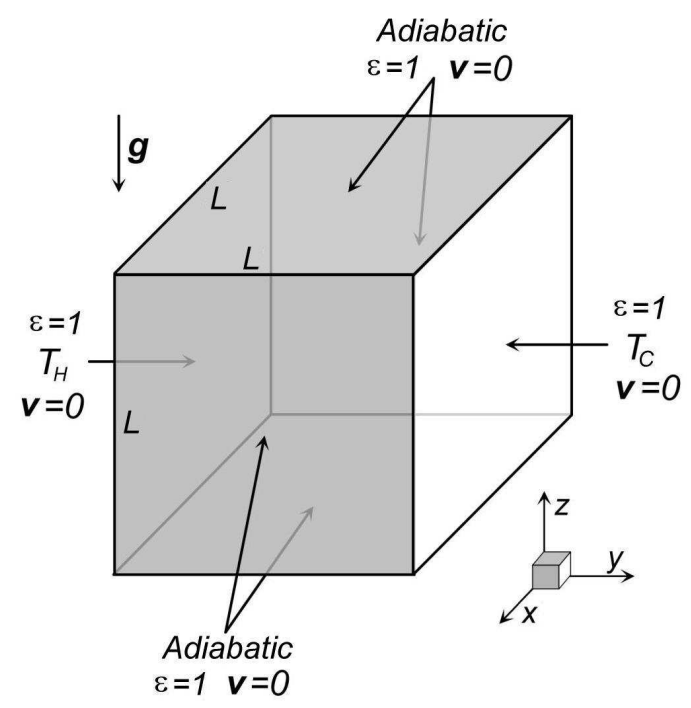

Figure 1. Three-dimensional differentially heated cavity scheme.

\section{MODELING}

We consider a Newtonian fluid whose flow, assumed to be laminar and steady state, is governed by the NSE obtained under the assumption of a low Mach number. The equation of state is that of an ideal gas and the transport equations are solved in their conservative form. The DOM is used to solve the RTE for a non-scattering medium, determining thus the radiation intensity field [e.g., Modest 2003]. To ensure the coupling between radiation and convection in the case of a participating medium, a source term is added in the energy equation. Under these assumptions, the energy equation can be written in the dimensional form as follows:

$$
\frac{\partial}{\partial x_{j}}\left(\rho T u_{j}\right)=\frac{\partial}{\partial x_{j}}\left(\frac{k_{0}}{C_{P 0}}\left(\frac{\partial T}{\partial x_{j}}\right)\right)-\nabla \cdot Q_{r a d}
$$

where the radiative dimensional divergence term is calculated as the difference between the emission and the absorption:

$$
\nabla \cdot Q_{\text {rad }}=\frac{\tau}{C_{P 0} L}\left(4 \sigma_{B} T^{4}-\int_{4 \pi} I d s\right)
$$

and variables $\rho, u_{j}, T$, and $I$ denote respectively the density, the velocity component along the $j$ direction, the temperature, and the radiation intensity (solution of the RTE). $k_{0}$ represents the thermal conductivity, $C_{P 0}$ is the specific heat at constant pressure, and $\sigma_{B}$ is the Stefan-Boltzmann constant. $\tau$ represents the optical thickness, defined by $\tau=\kappa \times L$ where $\kappa$ is the absorption coefficient. The RTE writes as follows:

$$
s \cdot \nabla I+\kappa I=\kappa \frac{\sigma_{B} T^{4}}{\pi}
$$

The radiation intensity depends both on space and directions. Physical properties of the RTE and several resolution methods are detailed in Modest's book [Modest 2003].

For the velocity field, no-slip boundary conditions are applied on the walls and for the temperature field uniform temperatures are imposed on the west and the east walls, as shown in Fig. 1. The 
adiabatic boundary condition applied on the remaining walls includes a conduction term and a radiation one. The temperature gradient at the adiabatic wall is given by:

$$
\frac{\partial T}{\partial n}=\frac{1}{k_{0}} Q_{\text {rad }} . n \quad \text { and } \quad Q_{\text {rad }} . n=\int_{4 \pi}(n \cdot s) I d s
$$

where $Q_{\text {rad. }} n$ represents the energy flux due to radiation through an arbitrarily oriented surface whose perpendicular vector is $n$, estimated with a weighted summation of the integrand at selected ordinates (directions) $s$. For non-participating media $(\tau=0)$, the coupling between the radiation transfer and the NSE is done via the radiative heat exchange between surfaces at the boundaries of the computational domain. Thus, radiation effects only depend on the temperature of the boundaries, as outlined in Colomer [2004]. Initially, we consider the fluid at rest, at a uniform temperature $T_{0}$, namely the mean value between $T_{h}$ and $T_{c}$.

The flow structure and the temperature distribution are governed, for a given optical thickness $\tau$, by the Rayleigh number $(R a)$, the Prandtl number $(P r)$, the Planck number $(P l)$, and the dimensionless temperature $T_{0}{ }^{*}$.

$$
R a=\frac{g \rho_{0}^{2} L^{3}\left(T_{h}-T_{c}\right) P r}{T_{0} \mu_{0}^{2}}, \quad \operatorname{Pr}=\frac{\mu_{0} C_{p 0}}{k_{0}}, \quad P l=\frac{k_{0}\left(T_{h}-T_{c}\right)}{L \sigma_{B} T_{c}^{4}}, \quad T_{0}^{*}=\frac{T_{c}}{\left(T_{h}-T_{c}\right)}
$$

Following the work of Colomer [2004], we consider air as the working fluid $\left(R=287 \mathrm{~J} . \mathrm{Kg}^{-1} . \mathrm{K}^{-1}\right.$, $\left.C_{P 0}=1004.5 \mathrm{~J} . \mathrm{Kg}^{-1} \cdot \mathrm{K}^{-1}, \mathrm{Pr}=0.71\right)$. The length of the cubic cavity $L$ was taken equal to $1 \mathrm{~m}$ and the thermodynamic pressure of reference $P_{t h 0}=1.013 \times 10^{5} \mathrm{~Pa}$. The present work is characterized by the governing parameters $R a=10^{6}$ and $P l=0.016$. The thermal conductivity obtained by $k_{0}=P l \sigma_{B} T_{c}^{4} L /\left(T_{h}-T_{c}\right)$ and the dynamical viscosity $\mu_{0}=k_{0} P r / C_{P 0}$ are constant. The gravity intensity $g=\operatorname{Ra} \mu_{0}^{2} T_{0} /\left(\rho_{0}^{2} \operatorname{PrL}^{3}\left(T_{h}-T_{c}\right)\right)$ is consistently modulated and the mean density value is obtained using the perfect gas law $\rho_{0}=P_{t h 0} / R T_{0}$. In the context of the low Mach number approximation, the mean temperature $T_{0}=\left(T_{h}+T_{c}\right) / 2$ is taken equal to $600 \mathrm{~K}$ and the mean temperature ratio $T_{0}{ }^{*}=1$, this leads to $T_{h}=800 \mathrm{~K}$ and to $T_{c}=400 \mathrm{~K}$. For the code validation, minor code modifications were necessary to perform the benchmark of Colomer [2004] with the same dimensionless control parameters, under the Boussinesq approximation. To deal with that, the mean temperature $T_{0}$ is taken equal to $300 \mathrm{~K}$, and the prescribed mean temperature ratio $\left(T_{0}{ }^{*}=17\right)$ leads to $T_{h}=308.5 \mathrm{~K}$ and $T_{c}=291.5 \mathrm{~K}$ approximately. The dimensionless local total heat flux at the hot wall can be calculated by:

$$
Q^{*}=\frac{1}{Q_{0}}\left(-k_{0} \frac{\partial T}{\partial n}+Q_{\text {rad }} \cdot n\right)_{y=0} \quad \text { with } \quad Q_{0}=\frac{k_{0}\left(T_{h}-T_{c}\right)}{L}
$$

where $Q_{0}$ represents the reference total heat flux. This total heat flux has been considered as the significant result to be presented for the three-dimensional differentially heated cavity solutions throughout this paper, as in the work of Colomer [2004].

\section{NUMERICAL METHOD}

The transport equations are solved by a fully-implicit finite volume method in a segregated formulation on a structured but non-uniform staggered mesh. The space discretization is based on high order schemes with flux limiters: QUICK scheme (third order scheme) is used for convection terms while diffusion terms are approached by a central difference approximation. The velocity-pressure coupling is treated using SIMPLER algorithm and the linearization of the equations relies on the Picard procedure. The linear systems obtained from the discretized transport equations are solved using the BiCGStab iterative method, while the linear system of the pressure equation (symmetric equation) is solved by the Conjugate Gradient (CG) method. The validity of the computation code has been checked on several benchmarks of natural and forced convection [e.g. Accary, to appear]. The 
use of under-relaxation techniques, when it was necessary, allowed a faster convergence and a better stability of the solution. A steady-state solution is supposed to be obtained when the residuals (in $L-\infty$ norm) of all transport equations reach $10^{-9}$ in dimensional form. Concerning the discrete ordinates contribution, the calculations were performed using the S8-approximation. All the results given throughout the paper were obtained with an upwind scheme for the interpolation of the radiation intensity, usually known as the Step scheme. The choice of weights and ordinates is similar to that of Modest [2003].

\section{CODE VALIDATION: THE BOUSSINESQ CASE}

The numerical code employed in this work has been verified by the means of the resolution of the benchmark problem treated in Colomer [2004]. The results have been briefly compared in order to validate the numerical method rather to give extensive numerical results. A quantitative agreement with the results was observed; this agreement required a non-uniform mesh of $N^{3}=100^{3}$ refined near the solid walls. Figure 2 shows the temperature field, where $T^{*}=\left(T-T_{c}\right) /\left(T_{h}-T_{c}\right)$, obtained in the context of Boussinesq approximation for a participating medium with a specified optical length $\tau=10$, and in which the governing parameters values are $R a=10^{6}, P l=0.016, T_{0}{ }^{*}=17$ and $\operatorname{Pr}=0.71$. A quick inspection shows that the solution presents symmetry with respect to the center of the cavity, which is a property of natural convection in the Boussinesq approximation for the considered configuration. We notice particularly that the temperature gradient at the adiabatic boundaries is not zero in the presence of radiation as mentioned in the Modeling section. For the same set of parameters, a study was performed to analyze the influence of the mesh spacing on the final result. Three different meshes, with $60^{3}, 80^{3}$, and $100^{3}$ control volumes were used. The convergence to an asymptotic solution can be observed by evaluating one or several pertinent quantities between one simulation and its previous coarser solution. Figure 3(a) shows the averaged (in the $z$-direction) dimensionless heat flux at the hot wall versus the depth $x$, for three grid sizes $\left(N=60^{3}, 80^{3}\right.$, and $\left.100^{3}\right)$. These results motivated the selection of the third mesh (i.e. the mesh with $100^{3}$ control volumes) as fine enough to perform the numerical studies. A comparison of the mean heat flux for three-dimensional situations has been also carried out for several optical thicknesses. Figure 3(b) shows the mean heat flux versus the depth $x$, for a non-participating medium $(\tau=0)$ and for a participating medium $(\tau=1$ and $\tau=10)$. Again a good agreement is obtained here with the results presented by Colomer [2004].

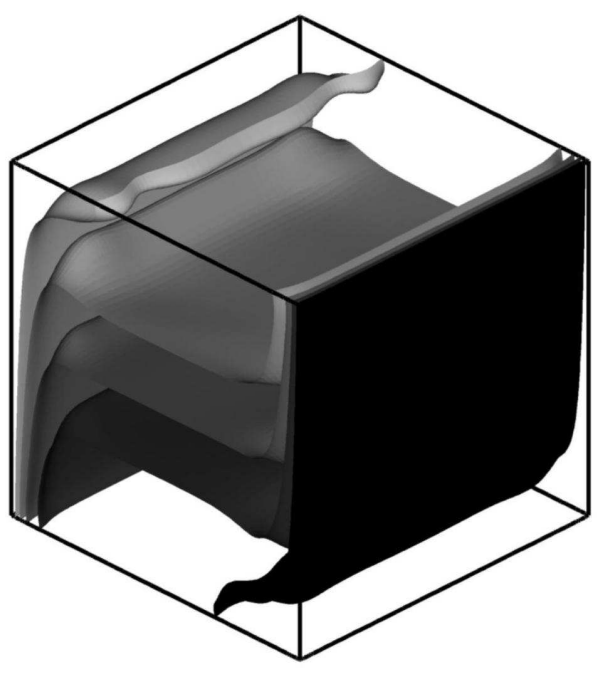

(a)

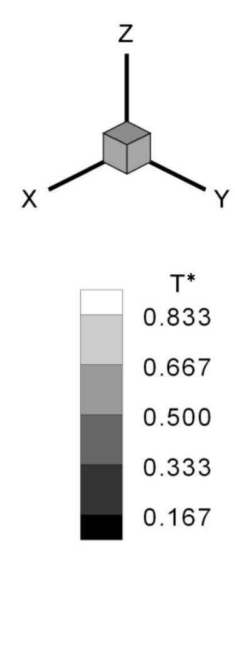

$\mathrm{T}^{*}$

667

.500

0.333

167

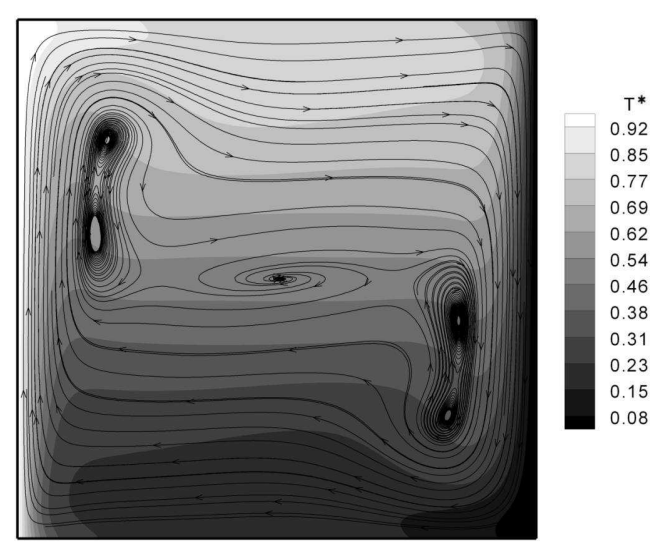

(b)

Figure 2. (a) Temperature field obtained in the Boussinesq approximation for $R a=10^{6}, P l=0.016$, $T_{0}{ }^{*}=17, \tau=10$, and $\operatorname{Pr}=0.71$. (b) A cut on the temperature field with the streamlines at $x=0.5$. 


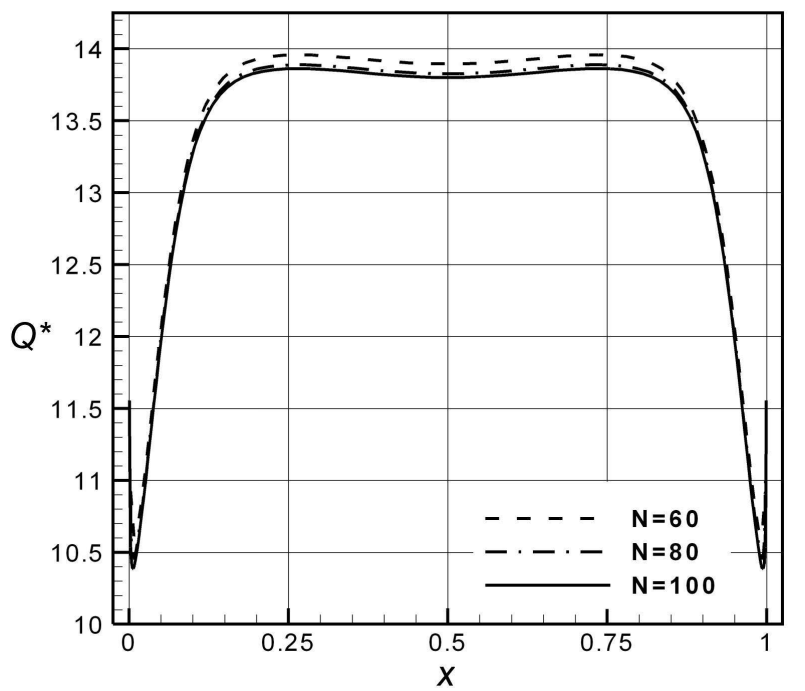

(a)

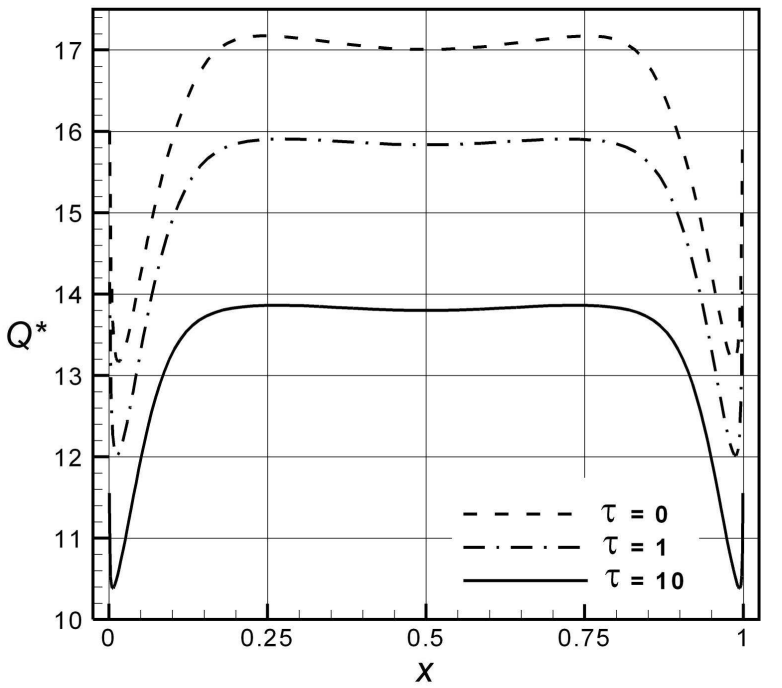

(b)

Figure 3. Average heat flux at the hot wall versus depth $\mathrm{x}$ obtained in the Boussinesq approximation for $R a=10^{6}, P l=0.016, T_{0}{ }^{*}=17, P r=0.71$. (a) For $\tau=10$ and for three grid sizes, showing the reaching of a mesh independent solution. (b) For $N=100$ and for $\tau=0,1,10$.

\section{EXTENSION TO THE LOW MACH NUMBER APPROXIMATION}

The investigation is now extended to a large temperature gap $\left(T_{h}-T_{c}\right)$ for which the Boussinesq assumption fails. Following the analysis done in the "code validation" section, figure 4 shows the temperature field, where $T^{*}=\left(T-T_{c}\right) /\left(T_{h}-T_{c}\right)$, obtained in the case of the low Mach number approximation for a participating medium with a specified optical length $\tau=10$, and in which the governing value parameters are $R a=10^{6}, \mathrm{Pl}=0.016, T_{0}{ }^{*}=1$ and $\operatorname{Pr}=0.71$. As expected we notice that the solution has lost its symmetry with respect to the center of the cavity, due to the fact that the density (computed from the ideal gas equation of state $\rho=P_{t h} / R T$ ) looses in this case its linear behaviour with respect to the temperature.

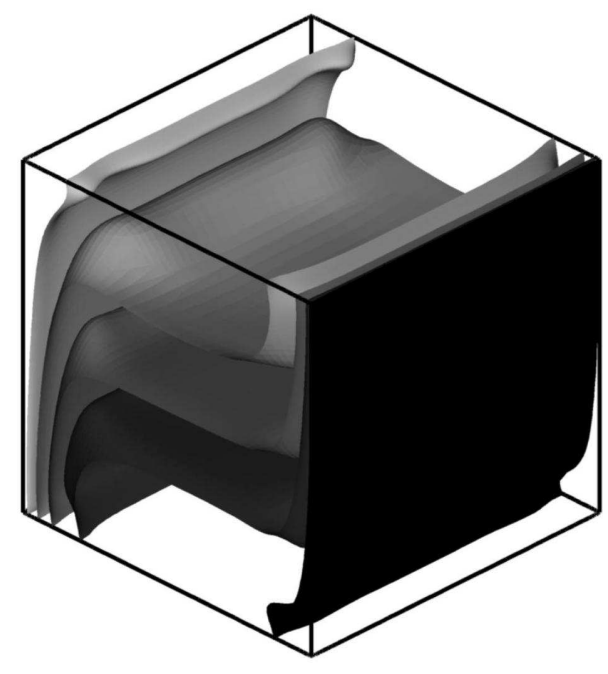

(a)

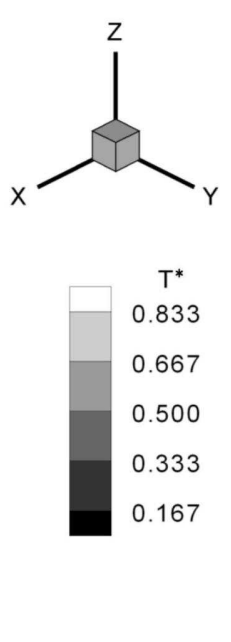

$\mathrm{T}^{*}$

0.667

.500

0.333

167

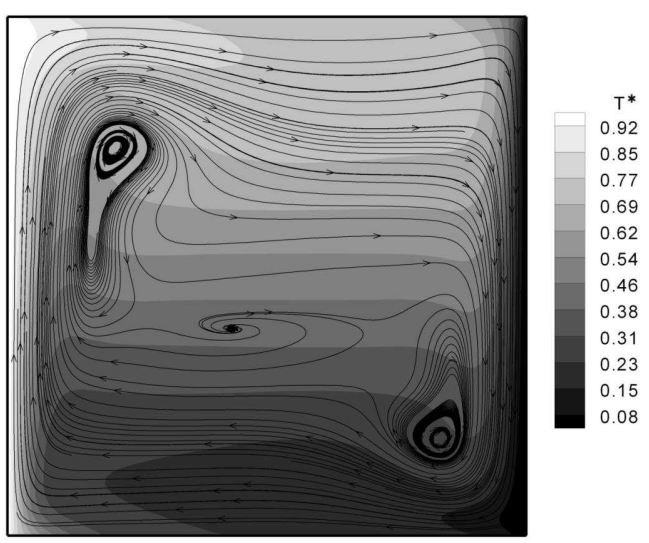

(b)

Figure 4. Temperature field obtained in the low Mach number approximation for $R a=10^{6}$, $P l=0.016, T_{0}{ }^{*}=1, \tau=10$, and $\operatorname{Pr}=0.71$. (b) A cut on the temperature field with the streamlines at $x=0.5$.

Similarly, a study was performed to analyze the influence of the mesh spacing on the final result. As done before, three different meshes, with $60^{3}, 80^{3}$ and $100^{3}$ control volumes were used. Figure 5(a) 
shows the averaged (in the $z$-direction) dimensionless heat flux at the hot wall versus the depth $x$, for three grid sizes $\left(N=60,80\right.$ and 100). Here also, the mesh with $100^{3}$ control volumes is fine enough to perform the numerical studies. A comparison of the mean heat flux for three-dimensional situations has been thus carried out for several values of the optical thickness. Figure 5(b) shows the mean heat flux versus the depth $x$, for a non-participating medium $(\tau=0)$ and for a participating medium ( $\tau=1$ and $\tau=10$ ). The mean heat fluxes at the boundaries obtained using the low Mach number approximation are 30 times larger (in dimensionless form) than those obtained in the Boussinesq approximation. This difference is only imputed to radiation; indeed without radiation, the mean Nusselt number $(\mathrm{Nu})$ at the hot wall, that can be obtained from the expression of $Q^{*}$ by dropping $Q_{r a d} \cdot n$, is nearly the same for both formulations at $R a=10^{6}$ and $\operatorname{Pr}=0.71 ; \mathrm{Nu}=8.63$ in the Boussinesq approximation and $N u=8.86$ in the low Mach number one [e.g. Accary 2006]. Finally, table 1 reports the thermodynamic pressures obtained in the low Mach number approximation for three optical thicknesses. The ratio of the thermodynamic pressures is given by:

$$
\frac{P_{t h}}{P_{t h 0}}=\frac{1}{\int_{\Omega} \frac{T_{0}}{T} d V}
$$

where $\Omega=[0,1] \times[0,1] \times[0,1]$ is the computation domain and $d V$ is a control volume. The fact that the thermodynamic pressure increases (resp. decreases) with decreasing (resp. increasing) $\tau$ indicates that the fluid volume is globally heated (resp. cooled).

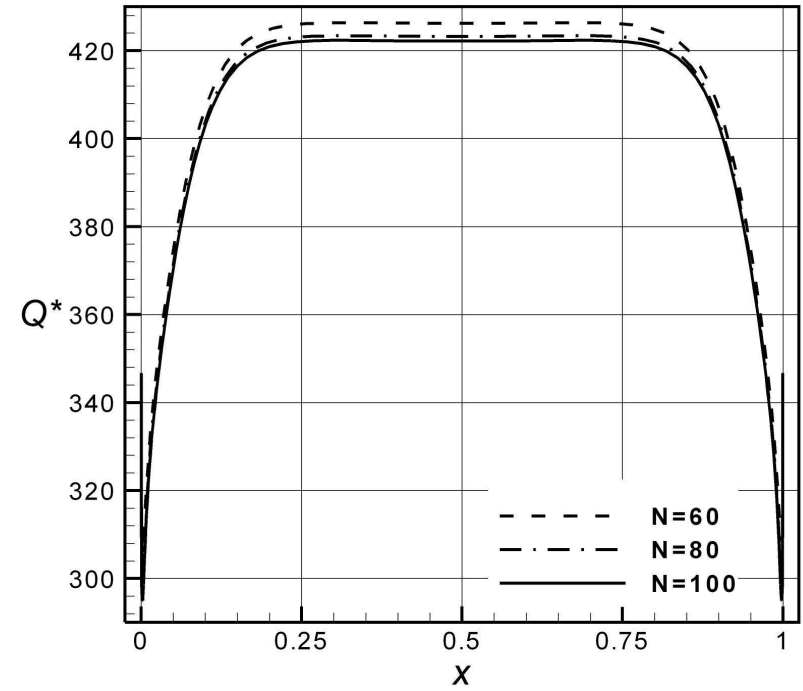

(a)

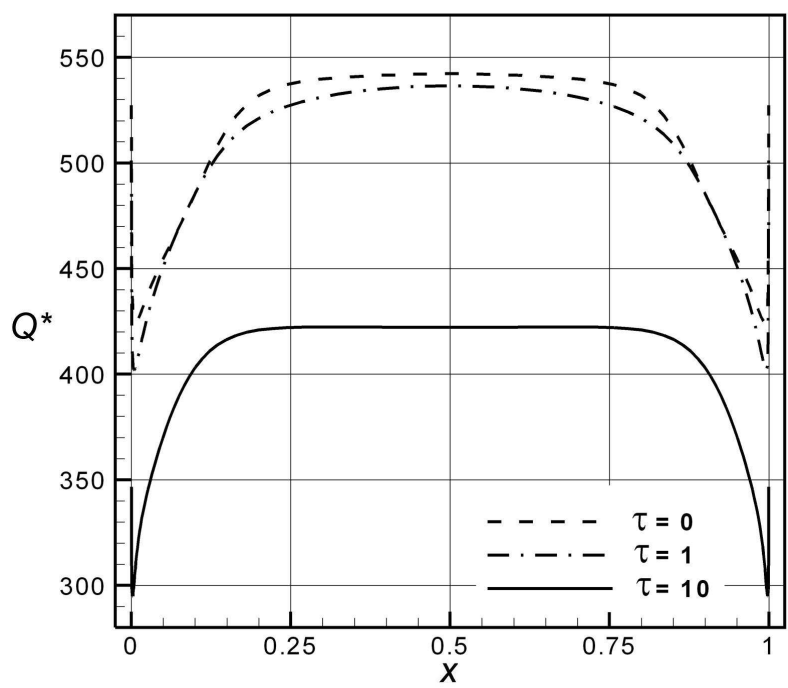

(b)

Figure 5. Average heat flux at the hot wall versus depth $\mathrm{x}$ obtained in the low Mach number approximation for $R a=10^{6}, \mathrm{Pl}=0.016, T_{0}{ }^{*}=1, \mathrm{Pr}=0.71$. (a) For $\tau=10$ and for three grid sizes, showing the reaching of a mesh independent solution. (b) For $N=100$ and for $\tau=0,1,10$.

\begin{tabular}{|l|c|c|c|}
\hline Optical thickness $\tau$ & 0 & 1 & 10 \\
\hline$P_{t h} / P_{\text {tho }}$ & 1.0732 & 1.0395 & 0.9764 \\
\hline
\end{tabular}

Table 1. Thermodynamic pressure obtained in the low Mach number approximation for $R a=10^{6}$, $P l=0.016, T_{0}{ }^{*}=1, P r=0.71$, and for $\tau=0,1,10$.

\section{CONCLUSION}

In this paper, the coupling between radiation and convection has been studied, for both transparent and participating (gray, purely absorbing) media, in a differentially heated cavity. The influence of the optical thickness has been studied for duly chosen values of Rayleigh and Planck numbers, both in the 
Boussinesq approximation and in the Low-Mach number one. It has been shown for both cases that a decrease of the optical thickness results in an increase in the heat flux at the isothermal boundaries. Important differences were observed between the two formulations, which justify the utility of proposed extension. In return, the authors are conscious that a particular effort should be made to overcome the well-known deficiencies of the DOM method, first by implementing more accurate discretization schemes [e.g., Coehlo 2002, Coelho 2002 (JCP)].

The investigation will be however extended to study the influence of the Rayleigh and the Planck numbers, and to take into account the presence of diffusive reflection and emission effects occurring at the walls $(\varepsilon \neq 1)$. This functionality is already implemented in the code, but for the sake of brevity, we restricted the present prospective work to the case $\varepsilon=1$. Another possible extension of the study is handling non-homogeneous, scattering and non-gray media in the DOM method. Finally, an OpenMP parallel version of the code is actually being developed and will soon be running on a SGI ALTIX cluster (consisting in 20 Itanium2 processors and $40 \mathrm{~GB}$ of shared memory). The angular decomposition strategy has been adopted for the parallelization of the DOM method, and has shown, in addition to its simplicity, higher efficiencies in comparison to an alternative approach based on spatial decomposition, [e.g, Gonçalves 1997].

\section{ACKNOWLEDGMENT}

The authors thank the European Community for the financial support in the context of the European integrated fire management project (Fire Paradox) aiming the modeling of the behavior of wildland fire.

\section{REFERENCES}

Accary, G., Meradji, S., Morvan, D., Fougère, D., Towards a Numerical Benchmark for 3D MixedConvection Low Mach Number Flows in a Rectangular Channel Heated from Below. Fluid Dynamics and Materials Processing, to appear.

Accary, G., Raspo, I. [2006], A 3D finite volume method for the prediction of a supercritical fluid buoyant flow in a differentially heated cavity. Comp. \& Fluids 35(10), 1316-1331.

Coehlo, P.J. [2002], The role of ray effects and false scattering on the accuracy of the standard and modified ordinates method, JSQRT, 73, 231-238.

Coelho, P.J. [2002], Bounded skew high-order resolution schemes for the Discrete Ordinates Method. J. Comp. Physics, 175, 412-437, 2002.

Coehlo, P.J. [2004], A modified version of the discrete ordinates method for radiative heat transfer modelling, Computational Mechanics, 33- 375-388.

Colomer, G., Costa, M., Consul, R., and Oliva, A. [2004], Three-dimensional numerical solution of convection and radiation in a differentially heated cavity using the discrete ordinates method, International Journal of Heat and Mass Transfer, 47, 357-269.

Gonçalves, J. and Coehlo [1997], P.J., Parallelization of the discrete ordinates method, Numer. Heat Trans. Part B, 32, 151-173.

Modest, M.F. [2003], Radiative heat transfer, Academic Press. 